\title{
Preference for Efficiency or Confusion? A Note on a Boundedly Rational Equilibrium Approach to Individual Contributions in a Public Good Game
}

\author{
Luca Corazzini ${ }^{1,2}$ and Marcelo Tyszler ${ }^{3}$ \\ ${ }^{1}$ Department of Law Science and History of Institutions, University of Messina, Piazza XX Settembre 4, \\ 98122 Messina, Italy \\ ${ }^{2}$ ISLA, Bocconi University, Via Roentgen 1, 20136 Milan, Italy \\ ${ }^{3}$ Center for Research in Experimental Economics and Political Decision Making (CREED), University of Amsterdam, \\ Roetersstraat 11, 1018 WB Amsterdam, Netherlands
}

Correspondence should be addressed to Marcelo Tyszler; marcelo@tyszler.com.br

Received 5 December 2014; Revised 25 March 2015; Accepted 29 March 2015

Academic Editor: Dimitris Fotakis

Copyright (C) 2015 L. Corazzini and M. Tyszler. This is an open access article distributed under the Creative Commons Attribution License, which permits unrestricted use, distribution, and reproduction in any medium, provided the original work is properly cited.

By using data from a voluntary contribution mechanism experiment with heterogeneous endowments and asymmetric information, we estimate a quantal response equilibrium (QRE) model to assess the relative importance of efficiency concerns versus noise in accounting for subjects overcontribution in public good games. In the benchmark specification, homogeneous agents, overcontribution is mainly explained by error and noise in behavior. Results change when we consider a more general QRE specification with cross-subject heterogeneity in concerns for (group) efficiency. In this case, we find that the majority of the subjects make contributions that are compatible with the hypothesis of preference for (group) efficiency. A likelihood-ratio test confirms the superiority of the more general specification of the QRE model over alternative specifications.

\section{Introduction}

Overcontribution in linear public good games represents one of the best documented and replicated regularities in experimental economics. The explanation of this apparently irrational behaviour, however, is still a debate in the literature. This paper is aimed at investigating the relative importance of noise versus preference for efficiency. In this respect, we build and estimate a quantal response equilibrium (henceforth, QRE [1]) extension of the model presented by Corazzini et al. [2]. This boundedly rational model formally incorporates both preference for efficiency and noise. Moreover, in contrast to other studies that investigate the relative importance of error and other-regarding preferences, the QRE approach explicitly applies an equilibrium analysis.
To reconcile the experimental evidence with the standard economic framework, social scientists developed explanations based on refinements of the hypothesis of "otherregarding preferences": reciprocity [3-6], altruism and spitefulness [7-9], commitment and Kantianism [10, 11], norm compliance [12], and team-thinking [13-15].

Recently, an additional psychological argument to explain agents' attitude to freely engage in prosocial behavior is gaining increasing interest: the hypothesis of preference for (group) efficiency. There is evidence showing that experimental subjects often make choices that increase group efficiency, even at the cost of sacrificing their own payoff $[16,17]$. Corazzini et al. [2] use this behavioral hypothesis to explain evidence from linear public good experiments based on prizes (a lottery, a first price all pay auction, and 
a voluntary contribution mechanism used as a benchmark), characterized by endowment heterogeneity and incomplete information on the distribution of incomes. In particular, they present a simple model in which subjects bear psychological costs from contributing less than what is efficient for the group. The main theoretical prediction of their model when applied to linear public good experiments is that the equilibrium contribution of a subject is increasing in both her endowment and the weight attached to the psychological costs of (group-) inefficient contributions in the utility function. The authors show that this model is capable of accounting for overcontribution as observed in their experiment, as well as evidence reported by related studies.

However, as argued by several scholars, rather than being related to subjects' kindness, overcontribution may reflect their natural propensity to make errors. There are several experimental studies [18-23] that seek to disentangle otherregarding preferences from pure noise in behavior by running ad hoc variants of the linear public good game. A general finding of these papers is that "warm-glow effects and random error played both important and significant roles" [20, p. 842] in explaining overcontribution.

There are several alternative theoretical frameworks that can be used to model noise in behavior (bounded rationality) and explain experimental evidence in strategic games. Two examples are the "level- $k$ " model (e.g., [24-26]) and (reinforcement) learning models (e.g., [27]). In the "level- $k$ " model of iterated dominance, "level-0" subjects choose an action randomly and with equal probability over the set of possible pure strategies while "level- $k$ " subjects choose the action that represents the best response against level- $(k-1)$ subjects. Level- $k$ models have been used to account for experimental results in games in which other-regarding preferences do not play any role, such as $p$-Beauty contests and other constant sum games. Since in public good games there is a strictly dominant strategy of no contribution, unless other-regarding preferences are explicitly assumed, "level- $k$ " models do not apply. Similar arguments apply to learning models. In the basic setting, each subject takes her initial choice randomly and with equal probability over the set of possible strategies. As repetition takes place, strategies that turn out to be more profitable are chosen with higher probability. Thus, unless other-regarding preferences are explicitly incorporated into the utility function, repetition leads to the Nash equilibrium of no contribution.

The QRE approach has the advantage that even in the absence of other-regarding preferences it can account for overcontribution in equilibrium. Moreover, we can use the model to assess the relative importance of noise versus efficiency concerns.

We start from a benchmark model in which the population is homogeneous in both concerns for (group) efficiency and the noise parameter. We then allow for heterogeneity across subjects by assuming the population to be partitioned into subgroups with different degrees of efficieny concerns but with the same value for the noise parameter.

In the QRE model with a homogeneous population, we find that subjects' overcontribution is entirely explained by noise in behavior, with the estimated parameter of concerns for (group) efficiency being zero. A likelihood-ratio test strongly rejects the specification not allowing for randomness in contributions in favor of the more general QRE model. A different picture emerges when heterogeneity is introduced in the QRE model. In the model with two subgroups, the probability of a subject being associated with a strictly positive degree of preference for (group) efficiency is approximately one-third. This probability increases to $59 \%$ when we add a third subgroup characterized by an even higher efficiency concern. A formal likelihood-ratio test confirms the superiority of the QRE model with three subgroups over the other specifications. These results are robust to learning processes over repetitions. Indeed, estimates remain qualitatively unchanged when we replicate our analysis on the last $25 \%$ of the experimental rounds. The rest of this paper is structured as follows. In Section 2, we describe the experimental setting. In Section 3, we present the QRE extension of the model based on the preference for (group) efficiency hypothesis. Section 4 reports results from our statistical analysis. Section 5 concludes the paper.

\section{The Experiment}

We use data from three sessions of a voluntary contribution mechanism reported by Corazzini et al. [2]. Each session consisted of 20 rounds and involved 16 subjects. At the beginning of each session, each subject was randomly and anonymously assigned, with equal chance, an endowment of either 120,160,200, or 240 tokens. The endowment was assigned at the beginning of the experiment and was kept constant throughout the 20 rounds. The experiment was run in a strangers condition [28] such that, at the beginning of each round, subjects were randomly and anonymously rematched in groups of four players. This procedure was common knowledge. Thus, in each round, subjects made their choices under incomplete information on the distribution of the endowments in their group. In each round, every subject had to allocate her endowment between an individual and a group account. The individual account implied a private benefit such that, for each token a subject allocated to the individual account, she received two tokens. On the other hand, tokens in the group account generated monetary returns to each of the group members. In particular, each subject received one token for each token allocated by her or by any other member of her group to the group account. Thus, the marginal per capita return used in the experiment was 0.5. At the beginning of each round, the experimenter exogenously allocated 120 tokens to the group account, independently of subjects' choices, thus implying 120 extra tokens for each group member. At the end of each round, subjects received information about their payoffs. Tokens were converted to euros using an exchange rate of 1000 points per euro. Subjects, mainly undergraduate students of economics, earned 12.25 euros on average for sessions lasting about 50 minutes. The experiment took place in May 2006 in the Experimental Economics Laboratory of the University 
of Milan Bicocca and was computerized using the $z$-Tree software [29].

The features of anonymity and random rematching narrow the relevance of some "traditional" behavioral hypotheses used to explain subjects' overcontribution. For instance, they preclude subjects' possibility to reciprocate (un)kind contributions of group members [30]. Moreover, under these conditions, subjects with preferences for equality cannot make compensating contributions to reduce (dis)advantageous inequality [31, 32]. Rather, the hypothesis of preference for (group) efficiency as a particular form of warm-glow $[8,9]$ appears as a more plausible justification.

\section{Theoretical Predictions and Estimation Procedure}

Consider a finite set of subjects $P=\{1,2, \ldots, p\}$. In a generic round, subject $i \in P$, with endowment $w_{i} \in N^{+}$, contributes $g_{i}$ to the group account, with $g_{i} \in N^{+}$and $0 \leq g_{i} \leq w_{i}$. The monetary payoff of subject $i$ who contributes $g_{i}$ in a round is given by

$$
\pi_{i}\left(w_{i}, g_{i}\right)=2\left(w_{i}-g_{i}\right)+120+g_{i}+G_{-i},
$$

where $G_{-i}$ is the sum of the contributions of group members other than $i$ in that round. Given (1), if subjects' utility only depends on the monetary payoff, zero contributions are the unique Nash equilibrium of each round. In order to explain the positive contributions observed in their experiment, Corazzini et al. [2] assume that subjects suffer psychological costs if they contribute less than what is optimal for the group. In particular, psychological costs are introduced as a convex quadratic function of the difference between a subject's endowment (i.e., the social optimum) and her contribution. In the VCM, player i's (psychological) utility function is given by

$$
u_{i}\left(w_{i}, g_{i}, \alpha_{i}\right)=\pi_{i}\left(w_{i}, g_{i}\right)-\alpha_{i} \frac{\left(w_{i}-g_{i}\right)^{2}}{w_{i}}
$$

where $\alpha_{i}$ is a nonnegative and finite parameter measuring the weight attached to the psychological costs, $\left(w_{i}-g_{i}\right)^{2} / w_{i}$, in the utility function. Notice that psychological costs are increasing in the difference between a subject's endowment and her contribution. Under these assumptions, in each round, there is a unique Nash equilibrium in which individual $i$ contributes:

$$
g_{i}^{\mathrm{NE}}=\frac{2 \alpha_{i}-1}{2 \alpha_{i}} w_{i} .
$$

The higher the value of $\alpha_{i}$, the higher the equilibrium contribution of subject $i$. The average relative contribution, $g_{i} / w_{i}$, observed in the VCM sessions is $22 \%$, which implies $\alpha=0.64$.

Following McKelvey and Palfrey [1], we introduce noisy decision-making and consider a Logit Quantal Response extension of (2). In particular, we assume subjects choose their contributions randomly according to a logistic quantal response function. Namely, for a given endowment, $w_{i}$, and contributions of the other group members, $G_{-i}$, the probability that subject $i$ contributes $g_{i}$ is given by

$$
q_{i}\left(w_{i}, g_{i}, \alpha_{i}, \mu\right)=\frac{\exp \left\{u_{i}\left(w_{i}, g_{i}, \alpha_{i}\right) / \mu\right\}}{\sum_{g_{j}=0}^{w_{i}} \exp \left\{u_{i}\left(w_{i}, g_{j}, \alpha_{i}\right) / \mu\right\}},
$$

where $\mu \in \mathfrak{R}_{+}$is a noise parameter reflecting a subject's capacity of noticing differences in expected payoffs.

Therefore each subject $i$ is associated with a $w_{i^{-}}$ dimensional vector $\underline{\mathbf{q}}_{i}\left(w_{i}, \underline{\mathbf{g}}_{i}, \alpha_{i}, \mu\right)$ containing a value of $q_{i}\left(w_{i}, g_{i}, \alpha_{i}, \mu\right)$ for each possible contribution level $g_{i} \in \underline{\mathbf{g}}_{i} \equiv$ $\left\{0, \ldots, w_{i}\right\}$. Let $\left\{\underline{\mathbf{q}}_{i}\left(w_{i}, \mathbf{g}_{i}, \alpha_{i}, \mu\right)\right\}_{i \in P}$ be the system including $\underline{\mathbf{q}}_{i}\left(w_{i}, \underline{\mathbf{g}}_{i}, \alpha_{i}, \mu\right), \forall i \in P$. Notice that since others' contribution, $G_{-i}$, enters the r.h.s of the system, others' $q_{i}$ will also enter the r.h.s. A fixed point of $\left\{\underline{\mathbf{q}}_{i}\left(w_{i}, \underline{\mathbf{g}}_{i}, \alpha_{i}, \mu\right)\right\}_{i \in P}$ is, hence, a quantal response equilibrium (QRE), $\left\{\underline{\mathbf{q}}_{i}^{\mathrm{QRE}}\left(w_{i}, \underline{\mathbf{g}}_{i}, \alpha_{i}, \mu\right)\right\}_{i \in P}$.

In equilibrium, the noise parameter $\mu$ reflects the dispersion of subjects' contributions around the Nash prediction expressed by (3). The higher the $\mu$, the higher the dispersion of contributions. As $\mu$ tends to infinity, contributions are randomly drawn from a uniform distribution defined over $\left[0, w_{i}\right]$. On the other hand, if $\mu$ is equal to 0 , the equilibrium contribution collapses to the Nash equilibrium. (more specifically, for each subject $i$ equilibrium contributions converge to $q_{i}\left(w_{i}, g_{i}^{\mathrm{NE}}, \alpha_{i}, 0\right)=1$ and $q_{i}\left(w_{i}, g_{i}, \alpha_{i}, 0\right)=0, \forall g_{i} \neq g_{i}^{\mathrm{NE}}$.)

In this framework, we use data from Corazzini et al. [2] to estimate $\alpha$ and $\mu$, jointly. We proceed as follows. Our initial analysis is conducted by using all rounds $(n=20)$ and assuming the population to be homogeneous in both $\alpha$ and $\mu$. This gives us a benchmark that can be directly compared with the results reported by Corazzini et al. [2]. In our estimation procedure, we use a likelihood function that assumes each subject's contributions to be drawn from a multinomial distribution. That is,

$$
L_{i}\left(w_{i}, \underline{\mathbf{g}}, i, \mu\right)=\frac{n !}{\prod_{g_{j}=0}^{w_{i}} n\left(g_{j}\right) !} \prod_{g_{k}=0}^{w_{i}} q_{i}^{\mathrm{QRE}}\left(w_{i}, g_{k}, \alpha, \mu\right)^{n\left(g_{k}\right)},
$$

where $n\left(g_{j}\right)$ is the number of times that subject $i$ contributed $g_{j}$ over the $n$ rounds of the experiment, and similarly for $n\left(g_{k}\right)$. The contribution of each person to the log-likelihood is the $\log$ of expression (5). The Maximum Likelihood procedure consists of finding the nonnegative values of $\mu$ and $\alpha$ (and corresponding QRE) that maximize the summation of the log-likelihood function evaluated at the experimental data. In other words, we calculate the multinomial probability of the observed data by restricting the theoretical probabilities to QRE probabilities only.

We then extend our analysis to allow for cross-subject heterogeneity. In particular, we generalize the QRE model above by assuming the population to be partitioned into $S$ 
TABLE 1: Homogeneous population (all rounds).

\begin{tabular}{lccccc}
\hline & Data & CFS & $(1) \bar{\mu}, \bar{\alpha}$ & $(2) \mu, \bar{\alpha}$ & $(3) \mu, \alpha$ \\
\hline$\mu$ & - & - & 1 & $21.83[19.69 ; 24.34]$ & $41.59[39.11 ; 44.34]$ \\
$\alpha$ & - & 0.64 & 0.64 & 0.64 & $0[0 ; 0.01]$ \\
\hline (Predicted) avg. contributions & & & & & \\
$\quad$ Overall endowments & 37.91 & 39.38 & 39.41 & 60.24 & 37.91 \\
$w_{i}=120$ & 34.02 & 26.25 & 26.34 & 44.84 & 34.12 \\
$w_{i}=160$ & 24.53 & 35.00 & 35.03 & 55.68 & 37.67 \\
$w_{i}=200$ & 47.50 & 43.75 & 43.76 & 65.57 & 39.48 \\
$w_{i}=240$ & 45.57 & 52.50 & 52.50 & 74.86 & 40.36 \\
\hline $\log l l$ & & & -8713.95 & -3483.79 & -3170.69 \\
Obs. & 960 & 960 & 960 & 960 & 960 \\
\hline
\end{tabular}

This table reports average contributions as well as estimates and predictions from various specifications of the model based on the efficiency concerns assumption using all 20 rounds of the experiment. CFS refers to the specification not accounting for noise in subjects' contributions while (1), (2), and (3) are Logit Quantal Response extensions of the model. In (1) $\alpha$ and $\mu$ are constrained to 0.64 and 1, respectively. In (2), the value on $\alpha$ is set to 0.64 , while $\mu$ is estimated through (5). Finally, (3) refers to the unconstrained model in which both $\alpha$ and $\mu$ are estimated through (5). The table also reports, for each specification, the corresponding log-likelihood. Confidence intervals are computed using an inversion of the likelihood-ratio statistic, at the 0.01 level, subject to parameter constraints.

subgroups that are characterized by the same $\mu$ but different $\alpha$. In this case, the likelihood function becomes

$$
\begin{aligned}
& L_{i}\left(w_{i}, \underline{\mathbf{g}}_{i}, \alpha_{1}, \alpha_{2}, \ldots, \alpha_{S}, \gamma_{1}, \gamma_{2}, \ldots, \gamma_{S}, \mu\right) \\
& =\sum_{s=1}^{S} \gamma_{s} \frac{n !}{\prod_{g_{j}=0}^{w_{i}} n\left(g_{j}\right) !} \prod_{g_{k}=0}^{w_{i}} q_{i}^{\mathrm{QRE}}\left(w_{i}, g_{k}, \alpha_{s}, \mu\right)^{n\left(g_{k}\right)},
\end{aligned}
$$

where $\gamma_{1}, \gamma_{2}, \ldots, \gamma_{S}$, with $\sum_{s=1}^{S} \gamma_{s}=1$, are the probabilities for agent $i$ belonging to the subgroup associated with $\alpha_{1}, \alpha_{2}, \ldots, \alpha_{S}$, respectively. This allows us to estimate the value of $\mu$ for the whole population, the value of $\alpha_{1}, \alpha_{2}, \ldots, \alpha_{S}$ for the $S$ subgroups, and the corresponding probabilities, $\gamma_{1}, \gamma_{2}, \ldots, \gamma_{S}$. For identification purposes we impose that $\alpha_{s} \leq$ $\alpha_{s+1}$. The introduction of one group at a time accompanied by a corresponding likelihood-ratio test allows us to determine the number of $\alpha$-groups that can be statistically identified from the original data. In the following statistical analysis, estimates account for potential dependency of subject's contributions across rounds. Confidence intervals at the 0.01 level are provided using the inversion of the likelihood-ratio statistic, subject to parameter constraints, in line with Cook and Weisberg [33], Cox and Hinkley [34], and Murphy [35].

\section{Results}

Using data from the 20 rounds of the experiment, Table 1 reports (i) average contributions (by both endowment type and overall) observed in the experiment, (ii) average contributions as predicted by the model not accounting for noise in subjects' contributions, and (iii) estimates as well as average contributions from different parameterizations of the Logit Quantal Response extension of the model. In particular, specification (1) refers to a version of the model in which both $\alpha$ and $\mu$ are constrained to be equal to benchmark values based on Corazzini et al. [2]. Under this parameterization, $\alpha$ is fixed to the value computed by calibrating (3) on the original experimental data, 0.64 , while $\mu$ is constrained to 1 . (Table 4 shows the Maximum Likelihood estimation value of $\alpha$ when we vary $\mu$. It is possible to see that for a large range of values of $\mu$ this value is close to 0.64 . We choose $\mu=1$ as a sufficiently low value in which the estimated $\alpha$ is close to 0.64 and thus provide a noisy version of the base model which can be used for statistical tests.)

As shown by the table, specification (1) closely replicates predictions of the original model presented by Corazzini et al. [2] not accounting for noise in subjects' contributions. In specification (2), $\alpha$ is fixed to 0.64 , while $\mu$ is estimated by using (4). The value of $\mu$ increases substantially with respect to the benchmark value used in specification (1). A likelihood-ratio test strongly rejects specification (1) that imposes restrictions on the values of both $\alpha$ and $\mu$ in favor of specification (2) in which $\mu$ can freely vary on $\mathfrak{R}_{+}$(LR $=$ 10460.33; $\left.\operatorname{Pr}\left\{\chi^{2}(1)>\operatorname{LR}\right\}<0.01\right)$. However, if we compare the predicted average contributions of the two specifications, we find that specification (1) better approximates the original experimental data. This is because a higher value of the noise parameter spread the distributions of contributions around the mean. Therefore even with mean contributions further from the data (induced by the fixed value of $\alpha$ ) the spread induced by the noise parameter in specification (2) produces a better fit. This highlights the importance of taking into account not only the average (point) predictions but also the spread around it. It also suggests that allowing $\alpha$ to vary can improve fit.

In specification (3), $\alpha$ and $\mu$ are jointly estimated using (5), subject to $\alpha \geq 0$. If both parameters can freely vary over $\mathfrak{R}_{+}, \alpha$ reduces to zero and $\mu$ reaches a value that is higher than what was obtained in specification (2). As confirmed by a likelihood-ratio test, specification (3) fits the experimental data better than both specification (1) 
TABLE 2: Homogeneous population (last 5 rounds).

\begin{tabular}{lccccc}
\hline & Data & CFS & $(1) \bar{\mu}, \bar{\alpha}$ & $(2) \mu, \bar{\alpha}$ & $(3) \mu, \alpha$ \\
\hline$\mu$ & & - & 1 & $11.63[9.80 ; 13.95]$ & $26.91[24.14 ; 30.17]$ \\
$\alpha$ & & 0.59 & 0.59 & 0.59 & $0[0 ; 0.03]$ \\
\hline (Predicted) avg. contributions & & & & \\
$\quad$ Overall endowments & 25.94 & 26.39 & 26.91 & 44.78 & 25.94 \\
$w_{i}=120$ & 21.13 & 17.59 & 18.44 & 34.55 & 25.05 \\
$w_{i}=160$ & 17.63 & 23.46 & 24.04 & 41.68 & 26.01 \\
$w_{i}=200$ & 35.28 & 29.32 & 29.71 & 48.30 & 26.39 \\
$w_{i}=240$ & 29.70 & 35.19 & 35.45 & 54.60 & -885.62 \\
\hline $\log l l$ & & & -1675.03 & -987.54 & 240 \\
Obs. & 240 & 240 & 240 & 240 & 240 \\
\hline
\end{tabular}

This table reports average contributions as well as estimates and predictions from various specifications of the model based on the efficiency concerns assumption using the last 5 rounds of the experiment only. The same remarks as in Table 1 apply.

TABLE 3: Heterogeneous subjects (all and last 5 rounds).

\begin{tabular}{lcccc}
\hline & $\mu, \alpha_{1}$, and $\alpha_{2}(n=20)$ & $\mu, \alpha_{1}$, and $\alpha_{2}(n=5)$ & $\mu, \alpha_{1}, \alpha_{2}$, and $\alpha_{3}(n=20)$ & $\mu, \alpha_{1}, \alpha_{2}$, and $\alpha_{3}(n=5)$ \\
\hline$\mu$ & $28.50[25.88 ; 31.26]$ & $15.07[12.90 ; 17.64]$ & $22.14[20.56 ; 23.95]$ & $14.25[12.04 ; 16.85]$ \\
$\alpha_{1}$ & $0[0 ; 0.01]$ & $0[0 ; 0.02]$ & $0[0 ; 0.01]$ & $0[0 ; 0.02]$ \\
$\alpha_{2}$ & $0.53[0.46 ; 0.60]$ & $0.54[0.47 ; 0.61]$ & $0.43[0.39 ; 0.46]$ & $0.48[0.40 ; 0.56]$ \\
$\alpha_{3}$ & & & $1.04[0.92 ; 1.16]$ & $0.76[0.53 ; 1.01]$ \\
$\gamma_{1}$ & $0.66[0.53 ; 0.78]$ & $0.63[0.50 ; 0.75]$ & $0.41[0.33 ; 0.46]$ & $0.59[0.49 ; 0.64]$ \\
$\gamma_{2}$ & & & $0.50[0.43 ; 0.55]$ & $0.34[0.23 ; 0.40]$ \\
\hline
\end{tabular}

\begin{tabular}{lcccc}
\hline (Predicted) avg. contributions & & & & \\
Overall endowments & 37.15 & 25.57 & 38.51 & 25.72 \\
$w_{i}=120$ & 32.06 & 22.17 & 31.97 & 22.07 \\
$w_{i}=160$ & 36.04 & 24.63 & 46.78 & 24.68 \\
$w_{i}=200$ & 39.01 & 26.77 & 40.83 & 26.99 \\
$w_{i}=240$ & 41.48 & 28.73 & -3083.35 & 29.13 \\
\hline $\log l l$ & -3112.06 & -865.75 & 960 & -865.16 \\
Obs. & 960 & 240 & 240 \\
\hline
\end{tabular}

This table reports estimates and predictions from two specifications of the model with efficiency concerns accounting for cross subject heterogeneity in the value of $\alpha$. The analysis is conducted both by including all experimental rounds and by focusing on the last five repetitions only. Parameters are estimated through (6). Given the linear restriction $\sum_{s=1}^{S} \gamma_{s}=1$, we only report estimates of $\gamma_{1}, \gamma_{2}, \ldots, \gamma_{S-1}$. Confidence intervals are computed using an inversion of the likelihood-ratio statistic, at the 0.01 level, subject to parameter constraints.

$\left(\mathrm{LR}=11086.54 ; \operatorname{Pr}\left\{\chi^{2}(2)>\mathrm{LR}\right\}<0.01\right)$ and specification (2) $\left(\mathrm{LR}=626.21 ; \operatorname{Pr}\left\{\chi^{2}(1)>\mathrm{LR}\right\}<0.01\right)$. Thus, under the maintained assumption of homogeneity, our estimates suggest that contributions are better explained by randomness in subjects' behavior rather than by concerns for efficiency.

In order to control for learning effects, we replicate our analysis using the last five rounds only.

Consistent with a learning argument, in both specifications (2) and (3), the values of $\mu$ are substantially lower than the corresponding estimates in Table 1 . Thus, repetition reduces randomness in subjects' contributions. The main results presented above are confirmed by our analysis on the last five periods. Looking at specification (3), in the model with no constraints on the parameters, the estimated value of $\alpha$ again drops to 0 . Also, according to a likelihoodratio test, specification (3) explains the data better than both specifications (1) $\left(\mathrm{LR}=1578.83 ; \operatorname{Pr}\left\{\chi^{2}(2)>\mathrm{LR}\right\}<0.01\right)$ and $(2)\left(\mathrm{LR}=203.85 ; \operatorname{Pr}\left\{\chi^{2}(1)>\mathrm{LR}\right\}<0.01\right)$.

These results seem to reject the preference for (group) efficiency hypothesis in favor of pure randomness in subjects' contributions. However, a different picture emerges when we allow for cross-subject heterogeneity. In Table 3 we drop the assumed homogeneity. We consider two models with heterogeneous subjects: the first assumes the population to be partitioned into two subgroups $(S=2)$ and the second into three subgroups $(S=3$ ). (We have also estimated a model with $S=4$. However, adding a fourth subgroup does not significantly improve the goodness of fit of the model compared to the specification with $S=3$. In particular, with $S=4$, the point estimates for the model with all periods are $\mu=21.81, \alpha_{1}=0, \alpha_{2}=0.38, \alpha_{3}=0.61, \alpha_{4}=1.04$, $\gamma_{1}=0.39, \gamma_{2}=0.42$, and $\gamma_{3}=0.09$.) As before, we conduct 
TABLE 4

\begin{tabular}{|c|c|c|}
\hline$\mu$ & $\alpha$ & Log-likelihood \\
\hline 1000.00 & 0 & -3637.64 \\
\hline 500.00 & 0 & -3591.79 \\
\hline 333.33 & 0 & -3548.7 \\
\hline 250.00 & 0 & -3508.34 \\
\hline 200.00 & 0 & -3470.67 \\
\hline 166.67 & 0 & -3435.64 \\
\hline 142.86 & 0 & -3403.19 \\
\hline 125.00 & 0 & -3373.25 \\
\hline 111.11 & 0 & -3345.76 \\
\hline 100.00 & 0 & -3320.64 \\
\hline 90.91 & 0 & -3297.8 \\
\hline 83.33 & 0 & -3277.17 \\
\hline 76.92 & 0 & -3258.65 \\
\hline 71.43 & 0 & -3242.16 \\
\hline 66.67 & 0 & -3227.61 \\
\hline 62.50 & 0 & -3214.93 \\
\hline 58.82 & 0 & -3204.01 \\
\hline 55.56 & 0 & -3194.79 \\
\hline 52.63 & 0 & -3187.18 \\
\hline 50.00 & 0 & -3181.1 \\
\hline 40.00 & 0 & -3171.22 \\
\hline 30.30 & 0.14 & -3192.52 \\
\hline 20.00 & 0.33 & -3247.22 \\
\hline 10.00 & 0.50 & -3444.42 \\
\hline 9.09 & 0.52 & -3488.26 \\
\hline 8.00 & 0.53 & -3555.57 \\
\hline 7.04 & 0.55 & -3634.45 \\
\hline 5.99 & 0.56 & -3753.67 \\
\hline 5.00 & 0.57 & -3916.89 \\
\hline 4.00 & 0.58 & -4173.35 \\
\hline 3.00 & 0.59 & -4615.92 \\
\hline 2.00 & 0.60 & -5547.35 \\
\hline 1.00 & 0.61 & -8506.13 \\
\hline 0.90 & 0.61 & -9181.67 \\
\hline 0.80 & 0.61 & -10032.07 \\
\hline 0.70 & 0.61 & -11133.38 \\
\hline 0.60 & 0.61 & -12612.63 \\
\hline 0.50 & 0.61 & -14699.13 \\
\hline 0.40 & 0.61 & -17852.64 \\
\hline
\end{tabular}

This table reports Maximum Likelihood estimates of $\alpha$ for selected values of $\mu$ (see (5)). The last column reports the corresponding log-likelihood value.

our analysis both by including all rounds of the experiment and by focusing on the last five repetitions only.

We find strong evidence in favor of subjects' heterogeneity. Focusing on the analysis over all rounds, according to the model with two subgroups, a subject is associated with $\alpha_{1}=0$ with probability 0.66 and with $\alpha_{2}=0.53$ with probability 0.34 . Results are even sharper in the model with three subgroups: in this case $\alpha_{1}=0$ and the two other $\alpha$ parameters are strictly positive: $\alpha_{2}=0.43$ and $\alpha_{3}=1.04$. Subjects are associated with these values with probabilities
$0.41,0.50$, and 0.09 , respectively. Thus, in the more parsimonious model, the majority of subjects contribute in a way that is compatible with the preference for (group) efficiency hypothesis. These proportions are in line with findings of previous studies $[18,21,22]$ in which, aside from confusion, social preferences explain the behavior of about half of the experimental population.

Allowing for heterogeneity across subjects reduces the estimated randomness in contributions: the value of $\mu$ reduces from 41.59 in specification (3) of the model with homogeneous population to 28.50 and 22.14 in the model with two and three subgroups, respectively. According to a likelihood-ratio test, both the models with $S=2$ and $S=3$ fit the data better than the (unconstrained) specification of the model with homogeneous subjects (for the model with $S=2$, $\mathrm{LR}=117.25 ; \operatorname{Pr}\left\{\chi^{2}(2)>\mathrm{LR}\right\}<0.01$, whereas for the model with $\left.S=3, \operatorname{LR}=174.66 ; \operatorname{Pr}\left\{\chi^{2}(4)>\operatorname{LR}\right\}<0.01\right)$. Moreover, adding an additional subgroup to the model, with $S=2$, significantly increases the goodness of fit of the specification $\left(\mathrm{LR}=57.42 ; \operatorname{Pr}\left\{\chi^{2}(2)>\mathrm{LR}\right\}<0.01\right)$. As before, all these results remain qualitatively unchanged when we control for learning processes and we focus on the last 5 experimental rounds.

In order to check for the robustness of our results in Table 3, we have also estimated additional specifications accounting for heterogeneity in both concerns for (group) efficiency and noise in subjects' behavior. Although the loglikelihood of the model with both sources of heterogeneity significantly improves in statistical terms, the estimated values of the $\alpha$-parameters remain qualitatively the same as those reported in the third column of Table 3.

\section{Conclusions}

Is overcontribution in linear public good experiments explained by subjects' preference for (group) efficiency or, rather, does it simply reflect their natural attitude to make errors? In order to answer this fundamental question, we estimate a quantal response equilibrium model in which, in choosing their contributions, subjects are influenced by both a genuine concern for (group) efficiency and a random noise in their behavior.

In line with other studies, we find that both concerns for (group) efficiency and noise in behavior play an important role in determining subjects' contributions. However, assessing which of these two behavioral hypotheses is more relevant in explaining contributions strongly depends on the degree of cross-subject heterogeneity admitted by the model. Indeed, by estimating a model with homogeneous subjects, the parameter capturing concerns for (group) efficiency vanishes while noise in behavior entirely accounts for overcontribution. A different picture emerges when we allow the subjects to be heterogeneous in their concerns for efficiency. By estimating a model in which the population is partitioned into three subgroups that differ in the degree of concerns for efficiency, we find that most of the subjects contribute in a way that is compatible with the preference for (group) efficiency hypothesis. A formal likelihood-ratio 
test confirms the supremacy of the QRE model with three subgroups over the other specifications.

Previous studies [18-23] tried to disentangle the effects of noise from other-regarding preferences by mainly manipulating the experimental design. Our approach adds a theoretical foundation in the form of an equilibrium analysis. In contrast to studies which focus mostly on (direct) altruism, we follow Corazzini et al. [2] and allow for preference for efficiency. Our results are in line with the literature in the sense that we also conclude that a combination of noise and social concerns plays a role. Our results, however, are directly supported by a sound theoretical framework proven valid in similar settings (e.g., [36]).

Recent studies [37, 38] have emphasized the importance of admitting heterogeneity in social preferences in order to better explain experimental evidence. In this paper we show that neglecting heterogeneity in subjects' social preferences may lead to erroneous conclusions on the relative importance of the love for (group) efficiency hypothesis with respect to the confusion argument. Indeed, as revealed by our analysis, the coupling of cross-subject heterogeneity in concerns for (group) efficiency with noise in the decision process seems to be the relevant connection to better explain subjects' contributions.

\section{Appendix}

Table 4 shows the Maximum Likelihood value of $\alpha$ and the log-likelihood according to (5) as $\mu$ decreases from 1000 to 0.4 . As shown by the table, for high values of $\mu$, the estimated value of $\alpha$ is 0 . When $\mu$ is equal to 10 , the estimated value of alpha is 0.50 . Moreover, for $\mu$ lower than 2.00 , the estimated value of $\alpha$ is 0.61 . For the specification tests presented in Section 4 , we set $\mu=1$. This is a sufficiently low value of $\mu$ in order to generate a noisy version of the base model. Two arguments indicate why this choice is valid. First, for a range of values including $\mu=1$, the estimated $\alpha$ is stable. Moreover, since the log-likelihood of a model with $\alpha=0.61$ and $\mu=1$ is higher than that corresponding to a model with $\mu=0.4$ (and similarly for $\alpha=0.64$ ), the choice of any $\mu$ lower than 1 for the benchmark value would only reinforce the results of Section 4. More specifically, both likelihood-ratio statistics comparing specifications (1) with specifications (2) and (3) of Tables 1 and 2 would increase.

\section{Conflict of Interests}

The authors declare that there is no conflict of interests regarding the publication of this paper.

\section{Acknowledgments}

The authors thank Arthur Schram, Jens Grosser, and participants to the 2010 Credexea Meeting at the University of Amsterdam, 2011 IMEBE in Barcelona, and 2011 Annual Meeting of the Royal Economic Society in London for useful comments and suggestions.

\section{References}

[1] R. D. McKelvey and T. R. Palfrey, "Quantal response equilibria for normal form games," Games and Economic Behavior, vol. 10, no. 1, pp. 6-38, 1995.

[2] L. Corazzini, M. Faravelli, and L. Stanca, "A prize to give for: an experiment on public good funding mechanisms," The Economic Journal, vol. 120, no. 547, pp. 944-967, 2010.

[3] R. Sugden, "Reciprocity: the supply of public goods through voluntary contributions," The Economic Journal, vol. 94, no. 376, pp. 772-787, 1984.

[4] H. Hollander, "A social exchange approach to voluntary contribution," The American Economic Review, vol. 80, no. 5, pp. 1157-1167, 1990.

[5] A. Falk and U. Fischbacher, "A theory of reciprocity," Games and Economic Behavior, vol. 54, no. 2, pp. 293-315, 2006.

[6] U. Fischbacher, S. Gächter, and E. Fehr, "Are people conditionally cooperative? Evidence from a public goods experiment," Economics Letters, vol. 71, no. 3, pp. 397-404, 2001.

[7] D. K. Levine, "Modeling altruism and spitefulness in experiments," Review of Economic Dynamics, vol. 1, no. 3, pp. 593-622, 1998.

[8] J. Andreoni, "Giving with impure altruism: applications to charity and ricardian equivalence," Journal of Political Economy, vol. 97, no. 6, pp. 1447-1458, 1989.

[9] J. Andreoni, "Impure altruism and donations to public goods: a theory of warm-glow giving," Economic Journal, vol. 100, no. 401, pp. 464-477, 1990.

[10] J. J. Laffont, "Macroeconomic constraints, economic efficiency and ethics: an introduction to Kantian economics," Economica, vol. 42, no. 168, pp. 430-437, 1975.

[11] M. Bordignon, "Was Kant right? Voluntary provision of public goods under the principle of unconditional commitment," Economic Notes, vol. 3, pp. 342-372, 1990.

[12] M. Bernasconi, L. Corazzini, and A. Marenzi, "Expressive' obligations in public good games: crowding-in and crowdingout effects," Working Papers, University of Venice "Ca' Foscari", Department of Economics, 2010.

[13] M. Bacharach, Beyond Individual Choice: Teams and Frames in Game Theory, Edited by N. Gold and R. Sugden, Princeton University Press, 2006.

[14] R. Sugden, “The logic of team reasoning," Philosophical Explorations, vol. 6, no. 3, pp. 165-181, 2003.

[15] R. Cookson, "Framing effects in public goods experiments," Experimental Economics, vol. 3, no. 1, pp. 55-79, 2000.

[16] G. Charness and M. Rabin, "Understanding social preferences with simple tests," Quarterly Journal of Economics, vol. 117, no. 3, pp. 817-869, 2002.

[17] D. Engelmann and M. Strobel, "Inequality aversion, efficiency, and maximin preferences in simple distribution experiments," American Economic Review, vol. 94, no. 4, pp. 857-869, 2004.

[18] J. Andreoni, "Cooperation in public-goods experiments: kindness or confusion?" The American Economic Review, vol. 85, no. 4, pp. 891-904, 1995.

[19] T. R. Palfrey and J. E. Prisbrey, "Altruism, reputation and noise in linear public goods experiments," Journal of Public Economics, vol. 61, no. 3, pp. 409-427, 1996.

[20] T. R. Palfrey and J. E. Prisbrey, "Anomalous behavior in public goods experiments: how much and why?" The American Economic Review, vol. 87, no. 5, pp. 829-846, 1997. 
[21] J. Brandts and A. Schram, "Cooperation and noise in public goods experiments: applying the contribution function approach," Journal of Public Economics, vol. 79, no. 2, pp. 399-427, 2001.

[22] D. Houser and R. Kurzban, "Revisiting kindness and confusion in public goods experiments," American Economic Review, vol. 92, no. 4, pp. 1062-1069, 2002.

[23] J. K. Goeree, C. A. Holt, and S. K. Laury, "Private costs and public benefits: unraveling the effects of altruism and noisy behavior," Journal of Public Economics, vol. 83, no. 2, pp. 255276, 2002.

[24] D. O. Stahl and P. W. Wilson, "On players' models of other players: theory and experimental evidence," Games and Economic Behavior, vol. 10, no. 1, pp. 218-254, 1995.

[25] T.-H. Ho, C. Cambrer, and K. Weigelt, "Iterated dominance and iterated best response in experimental 'p-Beauty Contests"' American Economic Review, vol. 88, no. 4, pp. 947-969, 1998.

[26] D. O. Stahl and E. Haruvy, "Level- $n$ bounded rationality in two-player two-stage games," Journal of Economic Behavior \& Organization, vol. 65, no. 1, pp. 41-61, 2008.

[27] I. Erev and A. E. Roth, "Predicting how people play games: reinforcement learning in experimental games with unique, mixed strategy equilibria," American Economic Review, vol. 88, no. 4, pp. 848-881, 1998.

[28] J. Andreoni, "Why free ride? Strategies and learning in public goods experiments," Journal of Public Economics, vol. 37, no. 3, pp. 291-304, 1988.

[29] U. Fischbacher, "Z-Tree: zurich toolbox for ready-made economic experiments," Experimental Economics, vol. 10, no. 2, pp. 171-178, 2007.

[30] M. Rabin, "Incorporating fairness into game theory and economics," The American Economic Review, vol. 83, no. 5, pp. 12811302, 1993.

[31] E. Fehr and K. M. Schmidt, "A theory of fairness, competition, and cooperation," Quarterly Journal of Economics, vol. 114, no. 3, pp. 817-868, 1999.

[32] G. E. Bolton and A. Ockenfels, "ERC: a theory of equity, reciprocity, and competition," American Economic Review, vol. 90, no. 1, pp. 166-193, 2000.

[33] R. D. Cook and S. Weisberg, "Confidence curves in nonlinear regression," Journal of the American Statistical Association, vol. 85, no. 410, pp. 544-551, 1990.

[34] D. R. Cox and D. V. Hinkley, Theoretical Statistics, Chapman and Hall, London, UK, 1974.

[35] S. A. Murphy, "Likelihood ratio-based confidence intervals in survival analysis," Journal of the American Statistical Association, vol. 90, no. 432, pp. 1399-1405, 1995.

[36] J. K. Goeree and C. A. Holt, "An explanation of anomalous behavior in models of political participation," American Political Science Review, vol. 99, no. 2, pp. 201-213, 2005.

[37] U. Fischbacher and S. Gachter, "Heterogeneous social preferences and the dynamics of free riding in public goods," CeDEx Discussion Paper 2006-01, 2006.

[38] M. Erlei, "Heterogeneous social preferences," Journal of Economic Behavior and Organization, vol. 65, no. 3-4, pp. 436-457, 2008. 


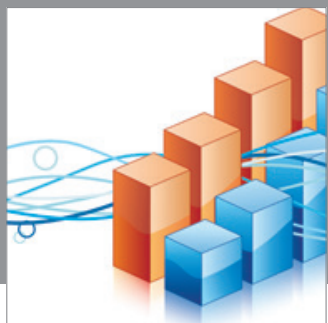

Advances in

Operations Research

mansans

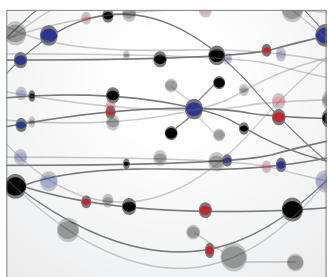

The Scientific World Journal
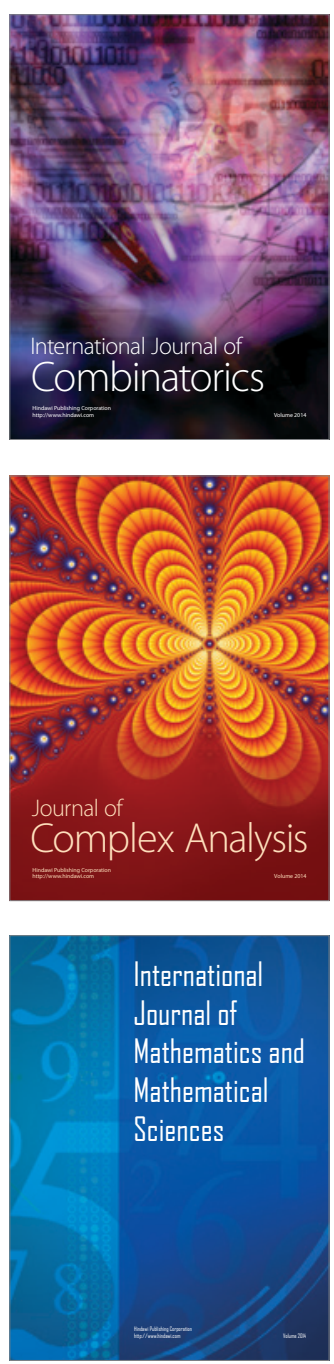
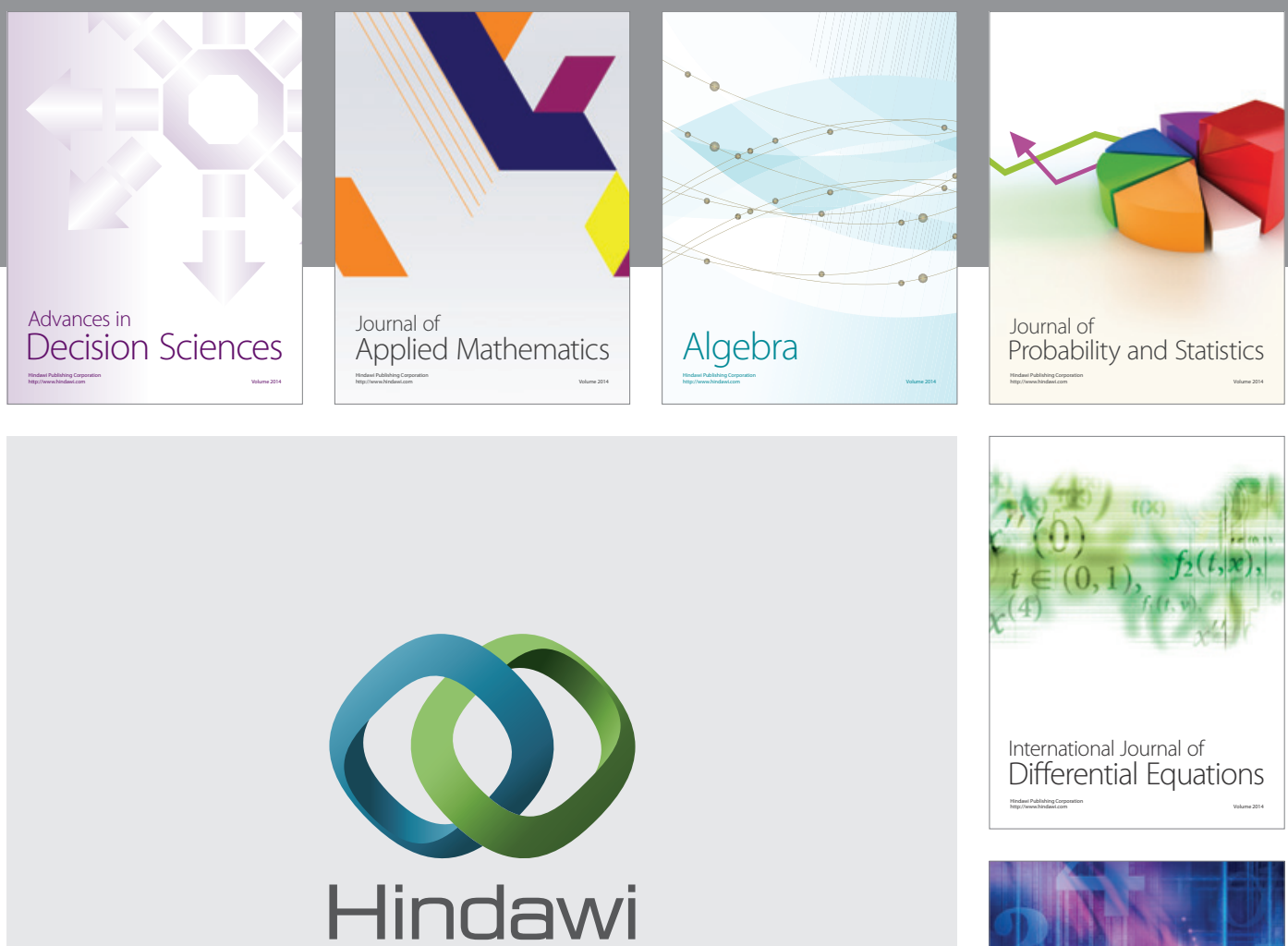

Submit your manuscripts at http://www.hindawi.com
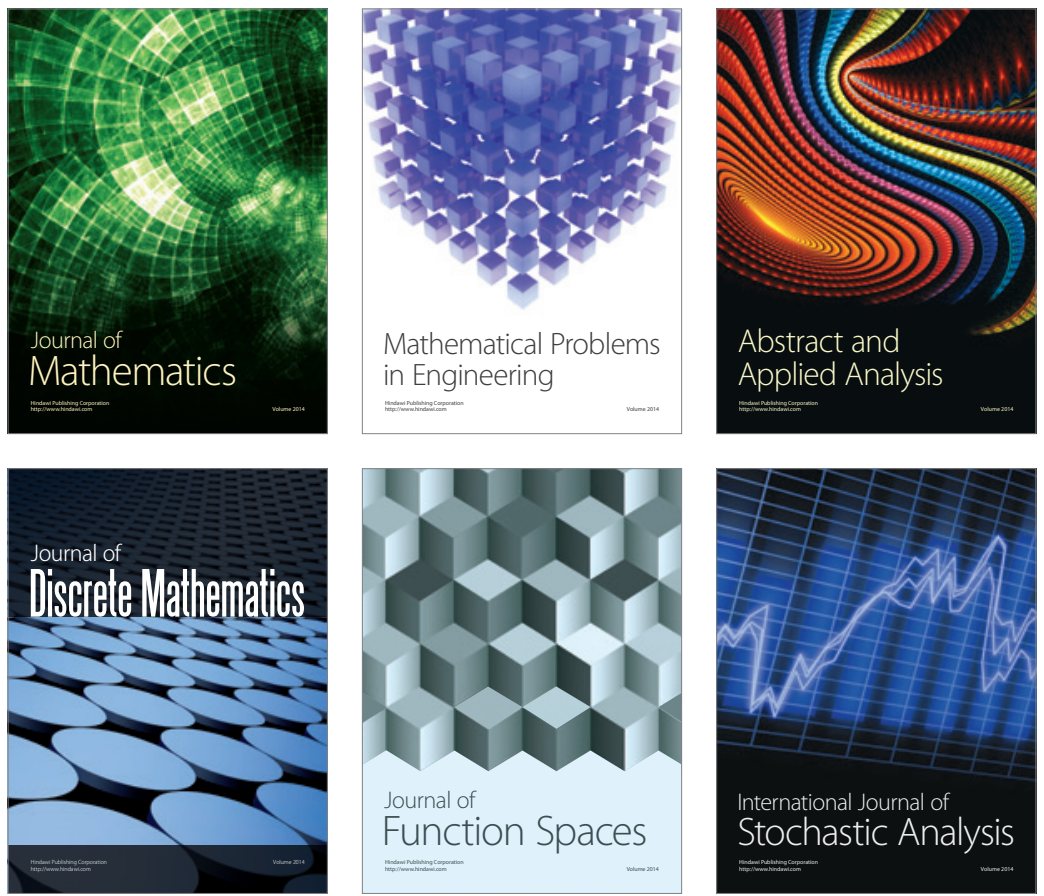

Journal of

Function Spaces

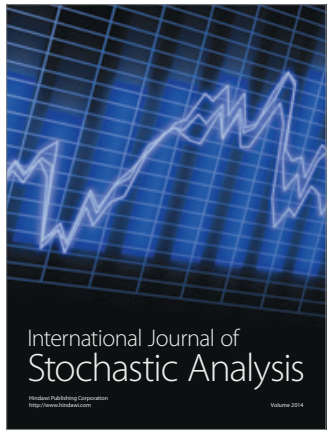

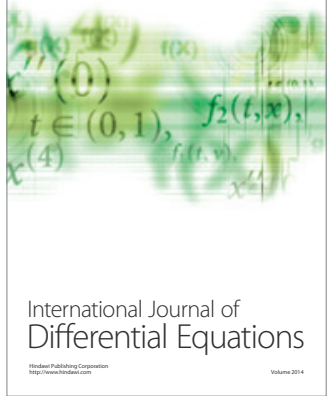
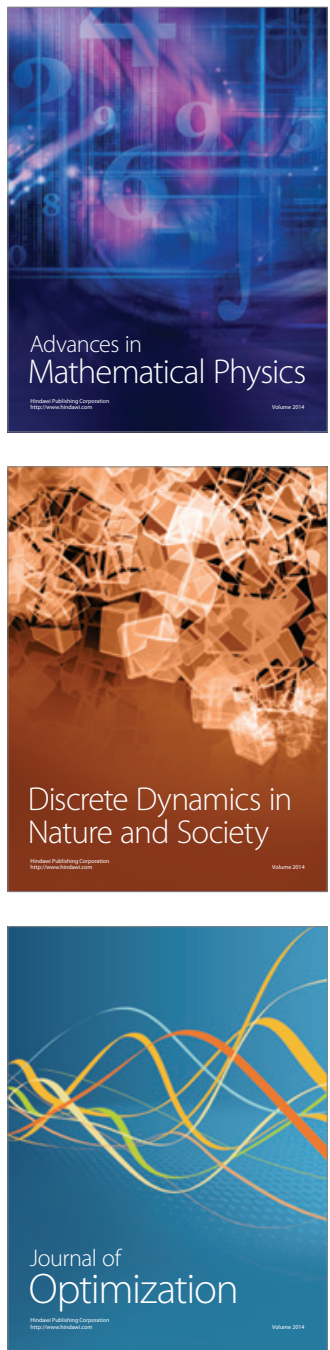